\title{
Identification of Enzymatic Active Sites with Unsupervised Language Modeling
}

\author{
Loïc Kwate Dassi \\ IBM Research Europe, Zürich \\ Daniel Probst \\ IBM Research Europe, Zürich \\ Yves Gaetan Nana Teukam \\ IBM Research Europe, Zürich
}

\author{
Matteo Manica \\ IBM Research Europe, Zürich \\ Philippe Schwaller \\ IBM Research Europe, Zürich \\ Teodoro Laino \\ IBM Research Europe, Zürich
}

\begin{abstract}
The first decade of genome sequencing saw a surge in the characterization of proteins with unknown functionality. Even still, more than $20 \%$ of proteins in well-studied model animals have yet to be identified, making the discovery of their active site one of biology's greatest puzzle. Herein, we apply a Transformer architecture to a language representation of bio-catalyzed chemical reactions to learn the signal at the base of the substrate-active site atomic interactions. The language representation comprises a reaction simplified molecular-input line-entry system (SMILES) for substrate and products, complemented with amino acid (AA) sequence information for the enzyme. We demonstrate that by creating a custom tokenizer and a score based on attention values, we can capture the substrateactive site interaction signal and utilize it to determine the active site position in unknown protein sequences, unraveling complicated 3D interactions using just $1 \mathrm{D}$ representations. This approach exhibits remarkable results and can recover, with no supervision, $31.51 \%$ of the active site when considering co-crystallized substrate-enzyme structures as a ground-truth, vastly outperforming approaches based on sequence similarities only. Our findings are further corroborated by docking simulations on the 3D structure of few enzymes. This work confirms the unprecedented impact of natural language processing and more specifically of the Transformer architecture on domain-specific languages, paving the way to effective solutions for protein functional characterization and bio-catalysis engineering.
\end{abstract}

\section{Introduction}

The number of anticipated genes has exploded as high-throughput sequencing technologies have improved dramatically. However, a considerable percentage of these newly found genes codifies proteins with no known function, which is a fundamental hindrance in our understanding of molecular life. The activity of a protein is directly related to the structure of the active site, a spatial region that, under external pressure, evolved its amino acids (AA) sequence to interact with specific molecules. The identification of active site residues and the characterization of the corresponding protein function remains a difficult task. While wet-lab experiments give the most accurate protein annotation, their limited throughput has increased the relevance of computational approaches. In fact, automated functional annotation is essential for understanding genomic data, which is essential to generate hypotheses about how proteins fit into processes and pathways. The last years have seen steady improvements in the field. Many top-performing algorithms frequently incorporate machine learning, 
and while a few are generally superior, no single method outperforms all the others [Jiang et al. 2016]. The most successful approaches for biological function annotation of proteins usually rely on 3D structural models [Yousaf et al., 2021, Sankararaman et al., 2010, Jiménez et al., 2017, Kozlovskii and Popov. 2021, Yang et al., 2013, Kozlovskii and Popov| 2020, Wass et al. 2010| to transfer functional insights, such as 3D features of protein-ligand binding sites, to the unknown proteins from structural homologies. More advanced schemes include sequence and protein-protein interaction network information to enhance the accuracy and coverage of the structure-based function predictions [Zhang et al. 2017]. There have also been a few efforts to identify active sites solely based on sequence similarity, such as PFAM [Mistry et al., 2020] and PSI-BLAST [Altschul et al., 1997].

In the last two years, BERT [Devlin et al., 2018], and more in general models pretrained via masked language modeling, have become the de-facto standard for different language understanding problems [Vig, 2019, Hoover et al., 2019, Rogers et al., 2020]. Self-supervised training techniques enabled the use of unlabeled and unstructured data sources to learn patterns in large corpora without requiring complex annotations. Their advent did not influence only the natural language domain but had an impact in multiple disciplines ranging from biology to chemistry, where string-based representations of proteins and molecules define a proteomic language [Rives et al., 2021, Elnaggar et al. 2020, Vig et al., 2020], respectively chemical language [Wang et al., 2019.|Kim et al., 2021], that can be used to interpret and understand physical phenomena. Unsupervised language models enabled the prediction of mutational effect and secondary structure, improving long-range contact prediction [Rives et al., 2021, Rao et al., 2019, Vig et al., 2020], the targeting of binding sites [Vig et al. 2020], the capture of important biophysical properties governing protein shape [Vig et al. 2020. Elnaggar et al. 2020]. For small molecules, Schwaller et al. [2021] showed how language models trained via masked language modeling (MLM) on organic chemical reaction data, represented using SMILES [Weininger, 1988], can leverage attention to efficiently and accurately map atoms between precursors and products with no supervision.

Language models are emerging as an effective strategy for learning very elusive signals from protein and molecule language representations [Öztürk et al. 2020]. The importance of structural features in active sites for enzyme function is reflected in the fact that the amino acids (AA) in the active site have been conserved more than the entire sequence during evolution. As a result, the signal (or pattern) describing the 3D interaction of amino acids with the corresponding target molecules in the active site may be a valuable source of data for function prediction using only sequences and no explicit 3D structural features.

Here, we approach the problem from a multi-modal angle to capture the signal between the active site amino acids and the atoms of the interacting molecules to identify a protein's active site. We extend the work of Schwaller et al. [2021] by developing a new method that employs a publicly available collection of enzymatic reactions. We use SMILES for modeling each interacting molecule combined with the AA sequence for the enzyme's fundamental structure. This approach can recover $31.51 \%$ of the active sites when considering co-crystallized substrate-enzyme structures as ground-truth with no supervision, largely outperforming current state-of-the-art methods relying only on sequence similarities.

\section{Methods}

We repurposed BERT [Devlin et al., 2018] by jointly training it with Masked Language Modeling (MLM) and a n-gram Masked Language Modeling [Xiao et al., 2020] (n-gram MLM, with $n=3$ ), leveraging Multi-task Transfer Learning (MTL) [Pesciullesi et al., 2020] on a combination of reaction SMILES representing organic reactions [Lowe, 2012] (weight assigned 0.1) and bio-catalyzed reactions [Probst et al. 2021] (weight assigned 0.9). For enzymatic reactions, each example consists of a reaction SMILES complemented with the AA sequence representation of the enzyme of interest (see Figure 1 for a depiction). As we train the model via MLM and n-gram MLM, we sparsely mask the reactants and the products and densely mask the enzyme sequence. To reduce the length of the resulting reactions [Filipavicius et al. [2020], we built a sub-word vocabulary for AA sequences using Byte Pair Encoding (BPE) [Gage, 1994] learned on UniProt [Consortium, 2020].

The prediction of the active regions of proteins is unsupervised and entirely based on the analysis of the attention values computed by the pretrained language model after encoding a reaction. If we label $S \in \mathbb{R}^{l \times d}(l=r+m+p)$ as the embedding of a given reaction and $r, m$, and $p$ refer to the length 


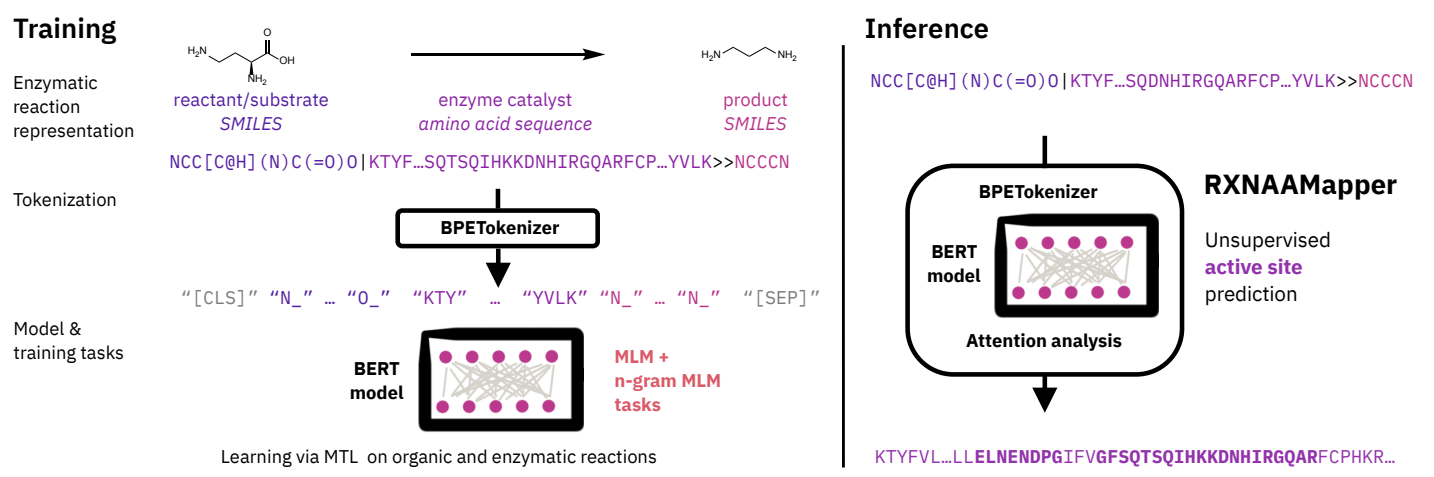

Figure 1: RXNAAMAPPER pipeline. A BERT model is trained on a combination of organic and enzymatic reaction SMILES using MTL, leveraging atom-level tokenization and MLM for the SMILES components, while BPE tokenization and n-gram MLM for the AA sequence part (left). The trained model is used in inference to define a score, based on the attention values computed on the reaction SMILES provided as input, which allows the prediction of the active site (bold-face AAs) of the enzyme bio-catalyzing the reaction with no supervision or structural information (right).

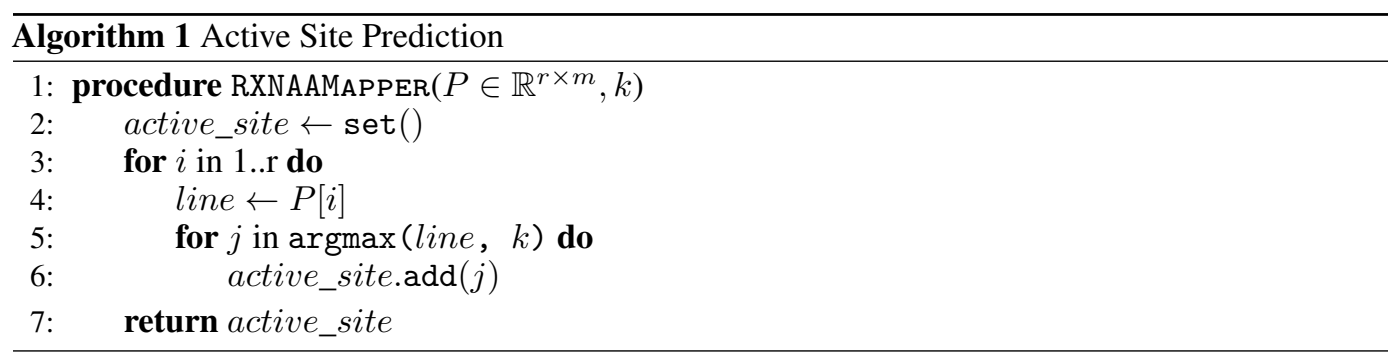

of the reactants, the enzyme, and the products, respectively, a forward pass of $S$ through the model yields a sequence $S^{\prime}$ with the same dimension as $S$. Each encoder block computes the attention matrix $A \in \mathbb{R}^{l \times l}$ of the sequence $S$ provided as input [Vaswani et al. 2017]. We construct a matrix $P \in \mathbb{R}^{r \times m}$ by summing two sub-matrices of $A$, describing the link between reactants and enzymes: $P=A[1: r, 1: m]+A[r+1: r+1+m, 1: r]^{T}$. We use the matrix $P$ as shown in the Algorithm 1 to predict the active regions via a consensus scheme where each reactant's atom has $k$ votes to choose its best-bound enzyme's token. The selected enzyme's tokens are uniquely gathered in a set and are considered the protein's active region. Hereinafter, the method will be referred to as RXNAAMapper.

\section{Evaluation}

We use a set of $5 \mathrm{~K}$ co-crystallized ligand-protein pairs from the Protein-Ligand Interaction Profiler (PLIP) [Salentin et al. 2015] as ground-truth, to perform a two-fold evaluation: (1) a sequence-based assessment benchmarking RXNAAMapper against a statistical baseline (Random Model), a pretrained BERT model on natural language (BERT-base) and the alignments retrieved from Pfam [Mistry et al. 2020]; and (2) a structural validation with protein-ligand binding energies computed with docking. We used PFAM for a fair assessment with existing methods using sequence information only. For the sequence-based evaluation, we use an overlap score between the prediction and the ground-truth, as well as the false positive rate. The overlap score is defined considering the active site as a set of non-overlapping segments in a sequence. If $S$ with $|S|=n$ is a sequence of amino acid residues, the active region $A_{s}$ of $S$ is defined as $A_{s}=\left\{\left(a_{i}, b_{i}\right)\right\}_{i}^{m}$, where $a_{i}$ and $b_{i}$ are the index boundaries of the segment $i$. The overlap score $\left(O S\left(A, A_{s}\right)\right)$ between the predicted active region $A=\left\{\left(a_{p i}, b_{p i}\right)\right\}_{i}^{n}$ and the ground-truth $A_{s}=\left\{\left(a_{s i}, b_{s i}\right)\right\}_{i}^{m}$ is defined as:

$$
O S\left(A, A_{s}\right)=\frac{\sum_{i}^{n} \sum_{j}^{m} \max \left(0, \min \left(b_{p i}, b_{s j}\right)-\max \left(a_{p i}, a_{s j}\right)\right)}{\sum_{i}^{m}\left(b_{s i}-a_{s i}\right)}
$$


Besides the overlap score, the false positive rate $(F P R)$ of the predictions is defined as:

$$
F P R=\frac{\sum_{i}^{n}\left(b_{p i}-a_{p i}\right) \mathbb{1} \bigwedge_{j=1}^{m}\left[a_{p i}, b_{p i}\right] \cap\left[a_{s j}, b_{s j}\right]=\emptyset}{\sum_{i}^{n}\left(b_{p i}-a_{p i}\right)}
$$

For the structural assessment, on a set of protein-ligand active sites predictions, we evaluated the binding energy computed with Autodock Vina [Eberhardt et al., 2021, Trott and Olson, 2010] considering predicted active sites and the ground-truth from PLIP.

\section{Experiments and Results}

In our experiments, we train models using a dataset of organic reactions from USPTO [Lowe 2012] ( $\sim 1 \mathrm{M}$, splits as reported in Schwaller et al. [2019]) combined with a dataset of bio-catalyzed reactions from ECREACT [Probst et al., 2021]. ECREACT records were further processed by using UniProt to replace and augment Enzyme Commission (EC) numbers with protein sequences from the corresponding EC classes. The resulting dataset has been further split into a training set $(\sim 7 \mathrm{M}$ reactions) and a validation set ( $\sim 4 \mathrm{~K}$ reactions). BERT has been trained with 4,094 as the batch size (leveraging gradient accumulation) and LAMB [You et al. 2020] as the optimization algorithm with 20,928 optimization steps.

Table 1 reports the performance of the different approaches on the PLIP ground-truth. Notably, RXNAAMapper performs consistently better than other methods, even though the product information has been completely omitted, given the nature of the dataset.

Table 1: Performance on sequence-based active site prediction. Reported in the table the overlap score and the false positive rates for the active site prediction using PLIP as a ground-truth for the four methods considered: a random model, Pfam alignment-based model, a pretrained BERT model and RXNAAMapper.

\begin{tabular}{lll}
\hline & Overlap Score & False Positive Rate \\
\hline Random Model & $4.98 \%$ & $84.20 \%$ \\
Pfam & $24.01 \%$ & $78.01 \%$ \\
BERT-base & $28.98 \%$ & $75.56 \%$ \\
RXNAAMapper (ours) & $\mathbf{3 1 . 5 1 \%}$ & $\mathbf{6 6 . 6 3 \%}$ \\
\hline
\end{tabular}

Figure 2a shows an exemplar comparison of Pfam-based and RXNAAMapper predictions overlapped with the PLIP ground-truth for a ligand-protein pair. Notably, RXNAAMapper controls the false positives better than Pfam alignments and matches the active site reported in PLIP.

(a)

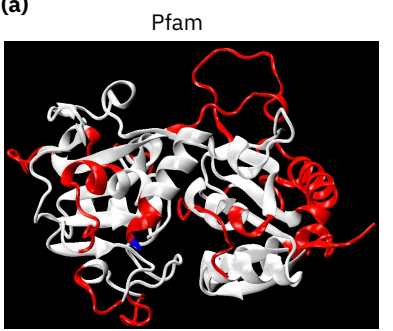

Background Prediction

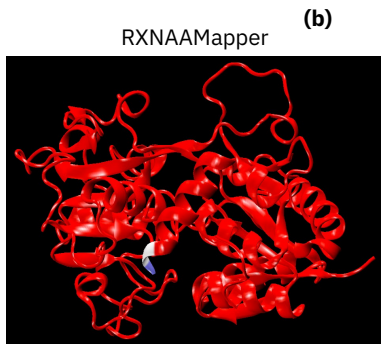

$\square$ Overlap

Ground-truth

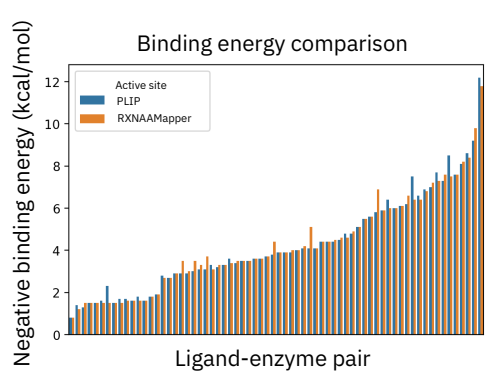

Figure 2: Experiment results. (a): comparison of the prediction from Pfam alignments (left) and RXNAAMapper (right) using PLIP as a ground-truth for an hydrolase (PDB id: 4FJP) interacting with zinc (SMILES: $[\mathrm{Zn}]$ ). (b): reported in the barplot the negative binding energy computed for a subset of protein-ligand pairs from PLIP using two configurations for the active site: experimental from PLIP (blue) and predicted via RXNAAMapper (orange).

For a subset of PLIP's protein-ligand pairs, we used Autodock Vina to compute the protein-ligand binding energy for the RXNAAMapper's predicted active sites and for the corresponding experimental ones provided by PLIP. We computed the binding energies by averaging the 3D coordinates of the 
active sites atoms and setting the box side length to $50 \AA$. In Figure $2 p$, we show the binding energies for the two active sites: RXNAAMapper and PLIP. We can appreciate how the binding energy calculations performed on the active site predicted by RXNAAMapper accurately match the one using the PLIP experimental information.

\section{Discussion}

The unsupervised detection of active sites using only protein sequence information is an important step to identify the function of uncharacterized proteins.

We presented RXNAAMapper, a technology that uses pretrained language models on text-based molecule representations to identify active sites in long amino acid sequences. Compared with protein-ligand interactions from PLIP, our approach outperforms other sequence-based baselines reconstructing $>30 \%$ of proteins' active regions while better controlling false positives. Furthermore, we have been able to validate the RXNAAMapper active sites predictions using docking studies by comparing the binding energy configurations with the experimental references.

These results show how large language models can inherently capture 3D structural information and reaction mechanisms from $1 \mathrm{D}$ representations, thus, opening up novel avenues for their consistent application in enzyme engineering.

\section{References}

Yuxiang Jiang, Tal Ronnen Oron, Wyatt T Clark, Asma R Bankapur, Daniel D'Andrea, Rosalba Lepore, Christopher S Funk, Indika Kahanda, Karin M Verspoor, and Asa et al. Ben-Hur. An expanded evaluation of protein function prediction methods shows an improvement in accuracy. Genome biology, 17(1):184, 2016.

Aqsa Yousaf, Tahira Shehzadi, Aqeel Farooq, and Komal Ilyas. Protein active site prediction for early drug discovery and designing. International Review of Applied Sciences and Engineering, 13(1):98 - 105, 2021. doi: 10.1556/1848.2021.00315. URL https://akjournals . com/view/ journals/1848/13/1/article-p98.xml.

Sriram Sankararaman, Fei Sha, Jack F Kirsch, Michael I Jordan, and Kimmen Sjölander. Active site prediction using evolutionary and structural information. Bioinformatics, 26(5):617-624, January 2010.

J Jiménez, S Doerr, G Martínez-Rosell, A S Rose, and G De Fabritiis. DeepSite: protein-binding site predictor using 3D-convolutional neural networks. Bioinformatics, 33(19):3036-3042, May 2017.

Igor Kozlovskii and Petr Popov. Protein-Peptide Binding Site Detection Using 3D Convolutional Neural Networks. Journal of chemical information and modeling, 61(8):3814-3823, August 2021.

Jianyi Yang, Ambrish Roy, and Yang Zhang. Protein-ligand binding site recognition using complementary binding-specific substructure comparison and sequence profile alignment. Bioinformatics, 29(20):2588-2595, August 2013.

Igor Kozlovskii and Petr Popov. Spatiotemporal identification of druggable binding sites using deep learning. Communications Biology, 3(1):618, 2020.

Mark N Wass, Lawrence A Kelley, and Michael J E Sternberg. 3DLigandSite: predicting ligandbinding sites using similar structures. Nucleic Acids Research, 38(2):W469-W473, May 2010.

Chengxin Zhang, Peter L. Freddolino, and Yang Zhang. COFACTOR: improved protein function prediction by combining structure, sequence and protein-protein interaction information. Nucleic Acids Research, 45(W1):W291-W299, 05 2017. ISSN 0305-1048. doi: 10.1093/nar/gkx366. URL https://doi.org/10.1093/nar/gkx366.

Jaina Mistry, Sara Chuguransky, Lowri Williams, Matloob Qureshi, Gustavo A Salazar, Erik L L Sonnhammer, Silvio C E Tosatto, Lisanna Paladin, Shriya Raj, Lorna J Richardson, Robert D Finn, and Alex Bateman. Pfam: The protein families database in 2021. Nucleic Acids Research, 49(D1):D412-D419, 10 2020. ISSN 0305-1048. doi: 10.1093/nar/gkaa913. URL https : //doi.org/10.1093/nar/gkaa913 
Stephen F. Altschul, Thomas L. Madden, Alejandro A. Schäffer, Jinghui Zhang, Zheng Zhang, Webb Miller, and David J. Lipman. Gapped BLAST and PSI-BLAST: a new generation of protein database search programs. Nucleic Acids Research, 25(17):3389-3402, 09 1997. ISSN 0305-1048. doi: 10.1093/nar/25.17.3389. URL/https://doi.org/10.1093/nar/25.17.3389

Jacob Devlin, Ming-Wei Chang, Kenton Lee, and Kristina Toutanova. Bert: Pre-training of deep bidirectional transformers for language understanding. arXiv preprint arXiv:1810.04805, 2018.

Jesse Vig. A multiscale visualization of attention in the transformer model. In Proceedings of the 57th Annual Meeting of the Association for Computational Linguistics: System Demonstrations, pages 37-42, 2019.

Benjamin Hoover, Hendrik Strobelt, and Sebastian Gehrmann. exbert: A visual analysis tool to explore learned representations in transformers models. arXiv preprint arXiv:1910.05276, 2019.

Anna Rogers, Olga Kovaleva, and Anna Rumshisky. A primer in bertology: What we know about how bert works. Transactions of the Association for Computational Linguistics, 8:842-866, 2020.

Alexander Rives, Joshua Meier, Tom Sercu, Siddharth Goyal, Zeming Lin, Jason Liu, Demi Guo, Myle Ott, C Lawrence Zitnick, Jerry Ma, et al. Biological structure and function emerge from scaling unsupervised learning to 250 million protein sequences. Proceedings of the National Academy of Sciences, 118(15), 2021.

Ahmed Elnaggar, Michael Heinzinger, Christian Dallago, Ghalia Rihawi, Yu Wang, Llion Jones, Tom Gibbs, Tamas Feher, Christoph Angerer, Martin Steinegger, et al. Prottrans: towards cracking the language of life's code through self-supervised deep learning and high performance computing. arXiv preprint arXiv:2007.06225, 2020.

Jesse Vig, Ali Madani, Lav R Varshney, Caiming Xiong, Nazneen Rajani, et al. Bertology meets biology: Interpreting attention in protein language models. In International Conference on Learning Representations, 2020.

Sheng Wang, Yuzhi Guo, Yuhong Wang, Hongmao Sun, and Junzhou Huang. Smiles-bert: large scale unsupervised pre-training for molecular property prediction. In Proceedings of the 10th ACM international conference on bioinformatics, computational biology and health informatics, pages 429-436, 2019.

Hyunseob Kim, Jeongcheol Lee, Sunil Ahn, and Jongsuk Ruth Lee. A merged molecular representation learning for molecular properties prediction with a web-based service. Scientific Reports, 11 (1):1-9, 2021.

Roshan Rao, Nicholas Bhattacharya, Neil Thomas, Yan Duan, Xi Chen, John Canny, Pieter Abbeel, and Yun S Song. Evaluating protein transfer learning with tape. Advances in neural information processing systems, 32:9689, 2019.

Philippe Schwaller, Benjamin Hoover, Jean-Louis Reymond, Hendrik Strobelt, and Teodoro Laino. Extraction of organic chemistry grammar from unsupervised learning of chemical reactions. Science Advances, 7(15):eabe4166, 2021.

David Weininger. Smiles, a chemical language and information system. 1. introduction to methodology and encoding rules. Journal of chemical information and computer sciences, 28(1):31-36, 1988.

Hakime Öztürk, Arzucan Özgür, Philippe Schwaller, Teodoro Laino, and Elif Ozkirimli. Exploring chemical space using natural language processing methodologies for drug discovery. Drug Discovery Today, 25(4):689-705, 2020. ISSN 1359-6446. doi: https://doi.org/ 10.1016/j.drudis.2020.01.020. URL https://www.sciencedirect.com/science/article/ pii/S1359644620300465.

Dongling Xiao, Yu-Kun Li, Han Zhang, Yu Sun, Hao Tian, Hua Wu, and Haifeng Wang. Ernie-gram: Pre-training with explicitly n-gram masked language modeling for natural language understanding. arXiv preprint arXiv:2010.12148, 2020. 
Giorgio Pesciullesi, Philippe Schwaller, Teodoro Laino, and Jean-Louis Reymond. Transfer learning enables the molecular transformer to predict regio-and stereoselective reactions on carbohydrates. Nature communications, 11(1):1-8, 2020.

Daniel Mark Lowe. Extraction of chemical structures and reactions from the literature. $\mathrm{PhD}$ thesis, University of Cambridge, 2012.

Daniel Probst, Matteo Manica, Yves Gaëtan Nana Teukam, Alessandro Castrogiovanni, Federico Paratore, and Teodoro Laino. Molecular transformer-aided biocatalysed synthesis planning. ChemRxiv, 2021. doi: 10.26434/chemrxiv.14639007. URL https://app.dimensions ai/details/publication/pub.1138314574andhttps://chemrxiv.org/engage/ api-gateway/chemrxiv/assets/orp/resource/item/60c75919842e6599a7db4990/ original/molecular-transformer-aided-biocatalysed-synthesis-planning.pdf.

Modestas Filipavicius, Matteo Manica, Joris Cadow, and Maria Rodriguez Martinez. Pre-training protein language models with label-agnostic binding pairs enhances performance in downstream tasks. arXiv preprint arXiv:2012.03084, 2020.

Philip Gage. A new algorithm for data compression. The C Users Journal archive, 12:23-38, 1994.

The UniProt Consortium. UniProt: the universal protein knowledgebase in 2021. Nucleic Acids Research, 49(D1):D480-D489, 11 2020. ISSN 0305-1048. doi: 10.1093/nar/gkaa1100. URL https://doi.org/10.1093/nar/gkaa1100

Ashish Vaswani, Shazeer Noam, Parmar Niki, Uszkoreit Jakob, Jones Llion, N. Gomez Aidan, Kaiser Lukasz, and Polosukhin Illia. Attention is all you need. CoRR, abs/1706.03762, 2017. URL http://arxiv.org/abs/1706.03762

Sebastian Salentin, Sven Schreiber, V Joachim Haupt, Melissa F Adasme, and Michael Schroeder. Plip: fully automated protein-ligand interaction profiler. Nucleic acids research, 43(W1):W443W447, 2015.

Jerome Eberhardt, Diogo Santos-Martins, Andreas F. Tillack, and Stefano Forli. Autodock vina 1.2.0: New docking methods, expanded force field, and python bindings. Journal of Chemical Information and Modeling, 61(8):3891-3898, 2021. doi: 10.1021/acs.jcim.1c00203. URL https: //doi.org/10.1021/acs.jcim.1c00203. PMID: 34278794.

Oleg Trott and A. Olson. Autodock vina: Improving the speed and accuracy of docking with a new scoring function, efficient optimization, and multithreading. Journal of Computational Chemistry, $31,2010$.

Philippe Schwaller, Teodoro Laino, Théophile Gaudin, Peter Bolgar, Christopher A Hunter, Costas Bekas, and Alpha A Lee. Molecular transformer: a model for uncertainty-calibrated chemical reaction prediction. ACS central science, 5(9):1572-1583, 2019.

Yang You, Jing Li, Sashank Reddi, Jonathan Hseu, Sanjiv Kumar, Srinadh Bhojanapalli, Xiaodan Song, James Demmel, Kurt Keutzer, and Cho-Jui Hsieh. Large batch optimization for deep learning: Training bert in 76 minutes, 2020. 